\title{
Efficacy of betahistine plus cognitive behavioral therapy on residual dizziness after successful canalith repositioning procedure for benign paroxysmal positional vertigo
}

This article was published in the following Dove Press journal:

Neuropsychiatric Disease and Treatment

\author{
Tian-ju Wan' \\ Yi-Chuan Yu' \\ Xiao-gang Zhao' \\ Ping Tang' \\ Yong-shu Gong ${ }^{2}$ \\ 'Department of Emergency, \\ Yongchuan Hospital of Chongqing \\ Medical University, Chongqing, China; \\ ${ }^{2}$ Department of Pediatrics, Yongchuan \\ Hospital of Chongqing Medical \\ University, Chongqing, China
}

Correspondence: Yong-shu Gong Department of Pediatrics, Yongchuan Hospital of Chongqing Medical University, 439 Xuanhua Road, Yongchuan, Chongqing 402160, China Tel/fax +86 234986 I I 20 Email gongyongshu@sohu.com
Background: Some patients still complain of residual dizziness after successful canalith repositioning procedure (CRP) for benign paroxysmal positional vertigo (BPPV). Previous study found that compared to the low-dose betahistine, the high-dose betahistine could yield better efficacy in treating residual dizziness. Therefore, this study was conducted to assess whether the addition of cognitive behavioral therapy (CBT) could make low-dose betahistine produce similar results to high-dose betahistine in treating residual dizziness.

Methods: The recruited patients were randomly assigned to receive either low-dose betahistine (6 mg/time, three times/day) or high-dose betahistine (12 mg/time, three times/day). Patients in the low-dose group also received CBT (twice a week, 1 hour per time). The treatment was continued for 4 weeks. The duration of residual dizziness, 25-item Dizziness Handicap Inventory (DHI), Hamilton Anxiety Rating Scale (HARS), and Hamilton Depression Rating Scale (HDRS) were recorded and analyzed. The duration of residual dizziness and DHI score were the primary outcomes, and the HARS and HDRS scores were the secondary outcomes.

Results: Each group had 50 patients. After treatment, the average DHI scores, HDRS scores, and HARS scores were significantly decreased in both groups. The duration of residual dizziness and average DHI score were nonsignificantly different $(P=0.08 ; P=0.06)$ between the two groups, although they were lower in the low-dose group. Compared to the high-dose group, the low-dose group had the significantly lower average HDRS score $(P=0.007)$ and HARS score $(P=0.02)$. Meanwhile, four patients in the high-dose group experienced intolerable stomach upset.

Conclusion: These results demonstrated that the addition of CBT could make low-dose betahistine produce similar results to high-dose betahistine in treating residual dizziness. Moreover, the low-dose betahistine plus CBT showed some advantages over high-dose betahistine in relieving depressive and anxiety symptoms and should be further explored.

Keywords: residual dizziness, benign paroxysmal positional vertigo, BPPV, cognitive behavioral therapy, CBT, betahistine

\section{Introduction}

Benign paroxysmal positional vertigo (BPPV) refers to the transient vertigo induced by the movement of the head to a specific position. ${ }^{1}$ Typically, each episode of vertigo continues $<1$ minute. BPPV is a disorder arising from the problem in the inner ear. Nowadays, it becomes the most common peripheral vestibular disease in clinical practice and ranks first among otolaryngology-related vertigo. ${ }^{2}$ Previous studies 
reported that the prevalence of BPPV could range from 10.7 to 64.0 cases per 100,000 population and the lifetime prevalence could be up to $2.1 \% .^{2,3} \mathrm{Up}$ to now, the specific cause of BPPV is still not identified, although cases may be associated with various disorders of the inner ear, a prolonged recumbent position or head trauma. ${ }^{4}$ The small calcified otolith moving around loosely in the inner ear may be involved in the underlying mechanism of this disease. Patients with BPPV usually have nausea and sometimes vomiting and are at increased risk for impairment in the performance of daily activities. ${ }^{5}$

BPPV could usually be resolved without special treatment. Anti-vertigo medications are not indicated in most cases, only if in acute, severe exacerbation of BPPV. A previous study reported that the median interval of days between the onset of symptoms and spontaneous resolution were 7 days and 17 days in untreated patients with affected horizontal canal and affected posterior canal, respectively. ${ }^{6}$ Actually, the canalith repositioning procedure (CRP) can be the first choice in the treatment of BPPV., ${ }^{7,8}$ This method has been widely promoted in clinical practice. CRP can help patients to get relief from BPPV by moving the otoconia to the part of inner ear where they will not cause dizziness. ${ }^{9}$ Successful CRP means that nystagmus (negative DixHallpike test results) and vertigo symptoms disappear after CRP. ${ }^{10,11}$ However, some patients with successful CRP for BPPV still report dizziness, which could last for a certain period. The causal factors of residual dizziness are still under debate: 1) Kim and Lee ${ }^{12}$ thought that the autonomic dysfunction might be a possible cause for residual dizziness; 2) canalith repositioning was not completely successful during CRP; then, the residual otolith debris could cause mild positional vertigo; ${ }^{13} 3$ ) more central adaptation was still needed after successful CRP, resulting in the recovery delay; ${ }^{14} 4$ ) anxiety/depressive symptoms might also be the important causes for residual dizziness. ${ }^{15}$

Residual dizziness could cause nervousness, panic, and insomnia and seriously affect the quality of life of patients. Many treatment methods have been developed to treat residual dizziness after successful CRP for BPPV. Jung et $\mathrm{al}^{15}$ reported that the anxiolytics (low-dose etizolam) could effectively reduce the residual dizziness. Deng et $\mathrm{al}^{16}$ found that Danhong injection, a traditional Chinese medicine, could significantly improve the residual dizziness. Ge et $a{ }^{17}$ found that the betahistine could effectively shorten the duration of residual dizziness. Another study reported that compared to the low-dose betahistine $(6 \mathrm{mg} /$ time, three times/day), the high-dose betahistine (12 mg/time, three times/day) could yield better efficacy in treating residual dizziness. ${ }^{18}$ However, the optimal treatment plan is still not identified.

Cognitive behavioral therapy (CBT) is a psychosocial intervention to improve mental health. It focuses on the development of personal coping strategies that target solving current problems and changing unhelpful patterns in cognitions, behaviors, and emotional regulation. ${ }^{19}$ In recent years, many researchers focus on the efficacy of CBT in treating patients with dizziness. ${ }^{20,21}$ Generally speaking, CBT is often recommended in combination with other therapy methods to treat health problems in clinical practice. Our previous study suggested that the addition of CBT could significantly reduce the dose of sertraline in treating persistent posturalperceptual dizziness (PPPD). ${ }^{22}$ Therefore, we hypothesized that the addition of CBT could make low-dose betahistine produce similar results to high-dose betahistine in treating residual dizziness after successful CRP for BPPV.

\section{Patients and methods}

\section{Patients and procedures}

Our study was reviewed and approved by the ethical committee of Chongqing Medical University (Chongqing, China) and was conducted in accordance with the Declaration of Helsinki. After a thorough physical examination and a review of clinical history, each candidate underwent the supine head-turning test or Dix-Hallpike test. In most cases (about 90\%), BPPV was involved with posterior semicircular canal, and most patients were diagnosed with canalithiasis type. ${ }^{23}$ Thus, only the canalithiasis type of BPPV patients involved with posterior semicircular canal were included in this study. The BPPV diagnosis was referred to the diagnostic criteria issued in 2006 by the Otorhinolaryngology Branch of Chinese Medical Association. ${ }^{24}$

The CRP described by Epley ${ }^{25}$ was conducted. We performed maneuver only one time for each patient. Patients with disappearance of nystagmus (negative Dix-Hallpike test results) and vertigo symptoms were recruited. Once such patients agreed to be the candidates for enrollment, they were told about the design and purpose of this study. Briefly, patients meeting the following criteria were included in this study: 1) diagnosis of canalithiasis type of BPPV involved with posterior semicircular canal; 2) positive symptoms of residual dizziness after successful CRP; 3) no terminal diseases, central diseases (including central vertigo), cerebrovascular diseases, vascular disorders, cranial traumas, or other systemic diseases; 4) no history of previous episodes of vertigo and no history of drug or alcohol abuse; and 5) did not 
receive concomitant therapy with anti-vertigo drugs, other antihistamines, thiazide diuretics, corticosteroids, calcium antagonists, antiaggregants, and benzodiazepines. The first patient was randomized in September 2015, and the last clinic visit occurred in March 2017. All patients provided written informed consent before treatment.

\section{Treatment methods}

The included patients were randomly assigned to receive either low-dose betahistine ( $6 \mathrm{mg} /$ time, three times/day) or high-dose betahistine (12 $\mathrm{mg} /$ time, three times/day). The randomization was performed using a random number sequence generated by computer. Meanwhile, patients in the low-dose group also received CBT (twice a week, 1 hour per time). The detailed procedure of CBT was described in our previous study. ${ }^{22}$ The main purpose of CBT was to make patients have a correct understanding on the occurrence of residual dizziness and establish confidence in overcoming it. This treatment was continued for 4 weeks.

\section{Measurement index}

Patients in both groups completed the residual dizziness survey at the first day after successful CRP and continued to do residual dizziness survey until the residual dizziness completely disappeared. The primary outcomes were the duration of residual dizziness and the 25-item Dizziness Handicap Inventory (DHI) score. The duration of residual dizziness was used to assess the efficacy of different treatment methods on improving the residual dizziness. The DHI score was used to help patients to rate the dizziness-related physical impairments, activity limitations, and restrictions in participation. ${ }^{26}$ The total score of DHI ranged from 0 (no disability) to 100 (severe disability). The secondary outcomes were the Hamilton Depression Rating Scale (HDRS; 17-item) score and Hamilton Anxiety Rating Scale (HARS) score. The HDRS and HARS scores were used to assess the depressive and anxiety symptoms of patients, respectively. ${ }^{22}$

\section{Statistical analyses}

Demographic variables were compared by Student's $t$-test and chi-squared test. The Student's $t$-test was used to compare the durations of residual dizziness between the two groups. The repeated-measures ANOVA was conducted to explore the group differences on DHI, HDRS, and HARS scores at four time points (at baseline and at weeks 1, 2, and 4). ${ }^{27}$ The criterion for statistical significance was set at $P<0.05$, and all analyses were conducted using SPSS 19.0 (IBM Corporation, Armonk, NY, USA).

\section{Results Demographic profile}

In total, 100 patients who met the abovementioned inclusion criteria were included and randomly assigned into the lowdose group $(n=50)$ and high-dose group $(n=50)$ at a ratio of 1:1. The baseline data of the included patients, such as age, body mass index (BMI), and depressive/anxiety symptoms, were similar between the two groups. The detailed information is described in Table 1. In the high-dose group, two patients after 1-week treatment and two patients after 2-week treatment withdrew from the study due to the intolerable stomach upset; four patients who stopped taking medicine were excluded after 2 -week treatment. In the low-dose group, one patient who stopped taking medicine and one patient who was unable to be contacted were excluded after 2-week treatment. The flow diagram is shown in Figure 1.

\section{Residual dizziness duration}

Residual dizziness after successful CRP was mainly characterized by lightheadedness (continuous or intermittent) or (and) intermittent unsteadiness. In the high-dose group, 20 patients, 11 patients, and 19 patients showed only lightheadedness, only intermittent unsteadiness, and both symptoms, respectively. In the low-dose group, 18 patients, 10 patients, and 22 patients showed only lightheadedness, only intermittent unsteadiness, and both symptoms, respectively. As shown in Figure 2, there was no significant difference in the duration of residual dizziness between the high-dose group (11.96 \pm 3.32 days) and low-dose group (10.80 \pm 3.41 days; $P=0.08$ ), although the low-dose group had a nonsignificantly lower duration of residual dizziness.

Table I Demographic profile of the recruited patients

\begin{tabular}{|c|c|c|c|}
\hline Variables & $\begin{array}{l}\text { High-dose } \\
\text { group }\end{array}$ & $\begin{array}{l}\text { Low-dose } \\
\text { group }\end{array}$ & $P$-value \\
\hline $\mathrm{n}$ & 50 & 50 & - \\
\hline Age (years) ${ }^{\mathrm{a}}$ & $52.14(9.70)$ & $52.86(9.01)$ & 0.70 \\
\hline Female/male ${ }^{b}$ & $29 / 21$ & $28 / 22$ & 0.84 \\
\hline Education (years) ${ }^{\mathrm{a}}$ & $7.02(2.97)$ & $7.14(3.02)$ & 0.84 \\
\hline BMI $\left(\mathrm{kg} / \mathrm{m}^{2}\right)^{\mathrm{a}}$ & $23.22(3.26)$ & $22.87(3.54)$ & 0.61 \\
\hline Smoking $(\mathrm{Y} / \mathrm{N})^{\mathrm{b}}$ & $14 / 36$ & $18 / 32$ & 0.39 \\
\hline Single household $(\mathrm{Y} / \mathrm{N})^{\mathrm{b}}$ & $10 / 40$ & $15 / 35$ & 0.25 \\
\hline DHI scores ${ }^{\mathrm{a}}$ & $52.4(7.58)$ & $53.3(8.15)$ & 0.57 \\
\hline HDRS scores ${ }^{\mathrm{a}}$ & $16.58(2.67)$ & $16.66(2.79)$ & 0.88 \\
\hline HARS scores ${ }^{\mathrm{a}}$ & I I.34 (2.52) & $11.14(3.39)$ & 0.74 \\
\hline
\end{tabular}

Notes: aMean \pm SD, $P$-value was from Student's $t$-test. ${ }^{b} P$-value was from chi-squared test.

Abbreviations: BMI, body mass index; DHI, 25-item Dizziness Handicap Inventory; HARS, Hamilton Anxiety Rating Scale; HDRS, Hamilton Depression Rating Scale; $\mathrm{N}$, no; Y, yes. 


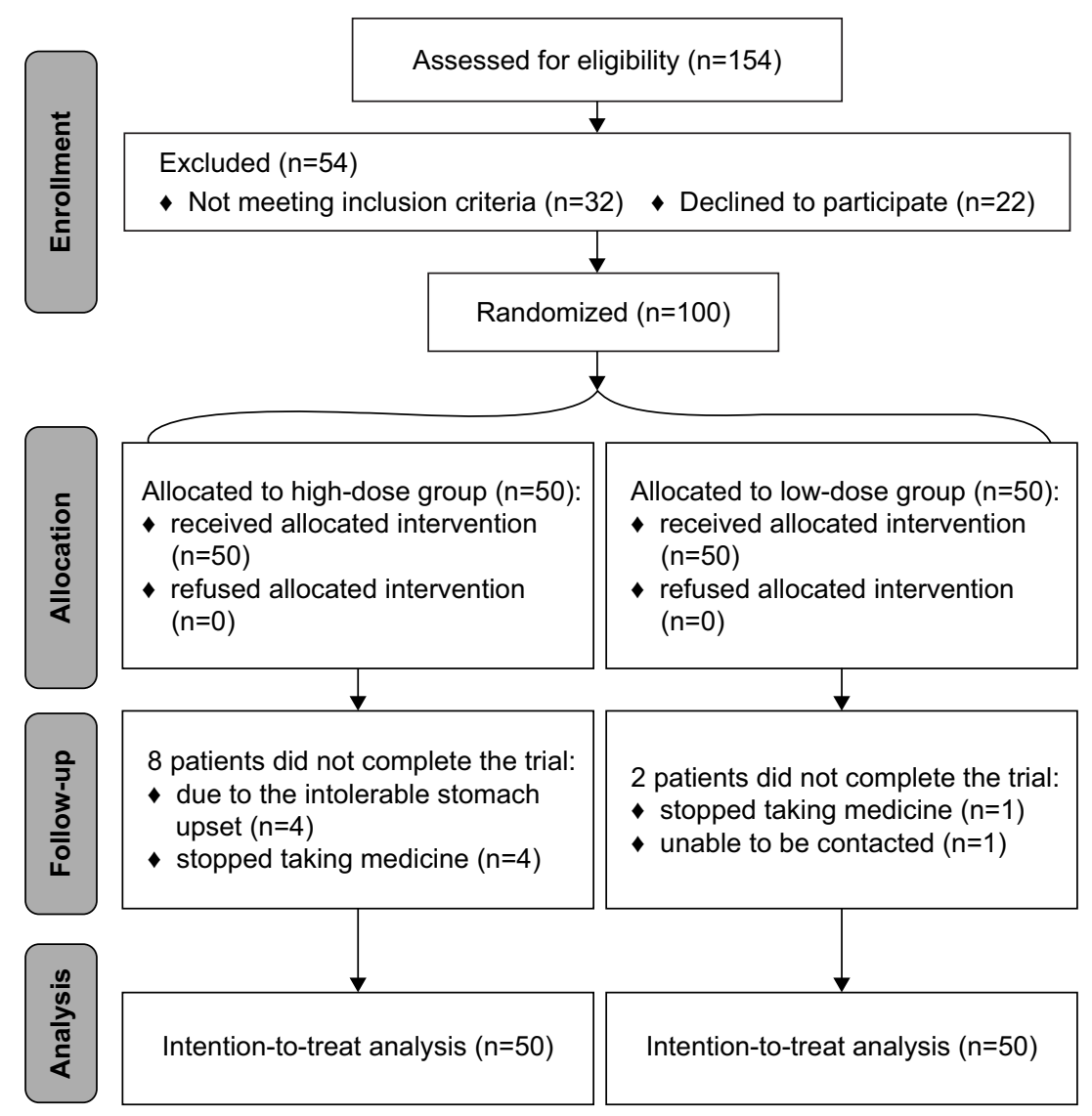

Figure I Flow diagram of this study.

\section{$\mathrm{DHI}$}

As shown in Figure 3, the average DHI scores decreased over time in both groups. After 4-week treatment, the average decrease in DHI score was similar between the two groups (26 points in the high-dose group and 29 points in the low-dose group, $P=0.06)$. The repeated-measures ANOVA showed a significant effect of time $(P<0.00001)$, indicating that both high-dose and low-dose betahistine could significantly reduce the DHI score. Meanwhile, the repeated-measures ANOVA showed no significant effect of group $\times$ time interaction $(P=0.17)$, which indicated that the reductions were nonsignificantly different between the two groups, although the low-dose group produced more reductions in DHI scores.

\section{Depressive symptoms}

As shown in Figure 4, the average HDRS scores decreased over time in both groups. After 4-week treatment, the average

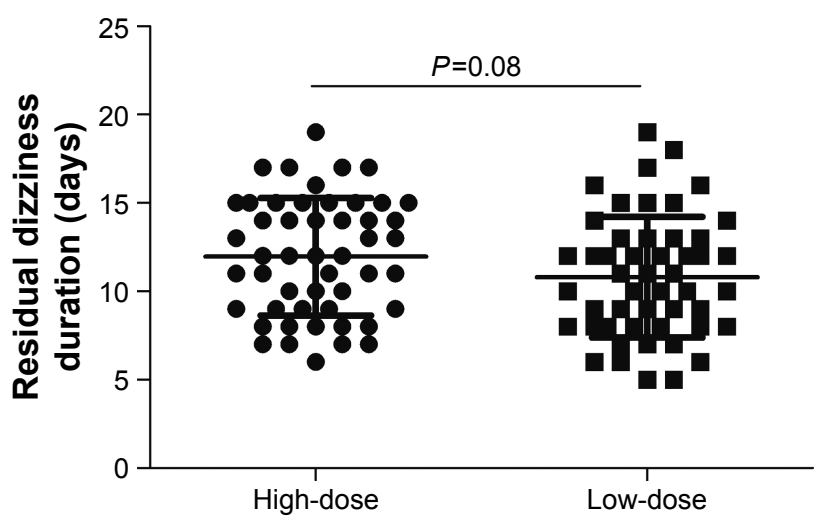

Figure 2 Duration of residual dizziness in both groups.

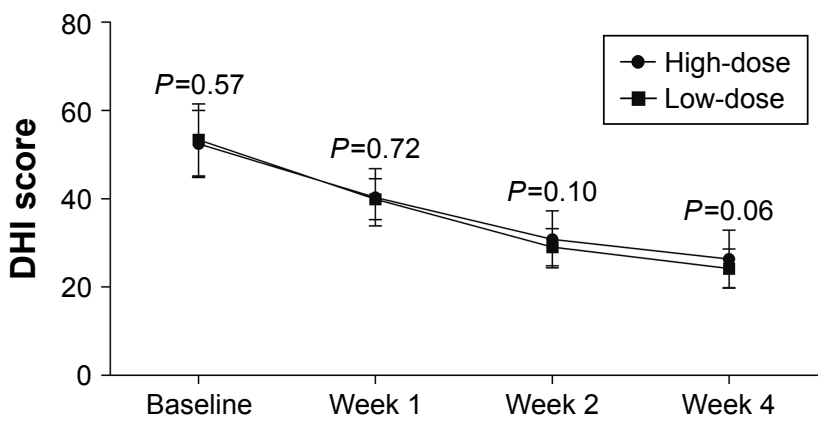

Figure 3 Average DHI scores at baseline and at weeks I, 2, and 4 in both groups. Abbreviation: DHI, 25-item Dizziness Handicap Inventory. 


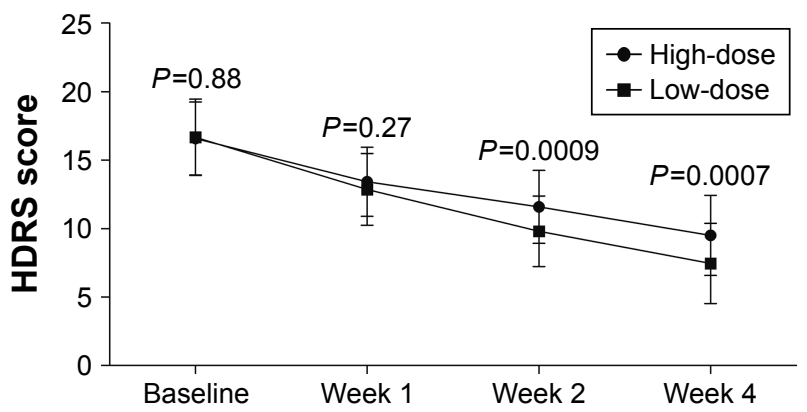

Figure 4 Average HDRS scores at baseline and weeks I, 2, and 4 in both groups. Abbreviation: HDRS, Hamilton Depression Rating Scale.

HDRS scores decreased from $16.58 \pm 2.67$ to $9.50 \pm 2.93$ in the high-dose group and from $16.66 \pm 2.79$ to $7.46 \pm 2.92$ in the low-dose group. The repeated-measures ANOVA showed a significant effect of time $(P<0.00001)$, indicating that both high-dose and low-dose betahistine could significantly reduce the HDRS score. Meanwhile, the repeated-measures ANOVA showed a significant effect of group $\times$ time interaction $(P=0.01)$, indicating the significantly different reductions between the two groups. The average HDRS scores were similar at baseline $(P=0.88)$ and week $1(P=0.27)$, but were significantly different at week $2(P=0.0009)$ and week 4 $(P=0.0007)$ between the two groups.

\section{Anxiety symptoms}

As shown in Figure 5, the average HARS scores decreased over time in both groups. After 4-week treatment, the average HARS scores decreased from $11.34 \pm 2.52$ to $5.46 \pm 2.14$ in the high-dose group and from $11.14 \pm 3.39$ to $4.38 \pm 2.55$ in the low-dose group. The repeated-measures ANOVA showed a significant effect of time $(P<0.00001)$, indicating that both high-dose and low-dose betahistine could significantly reduce the HARS score. Meanwhile, the repeated-measures ANOVA showed a significant effect of group $\times$ time interaction $(P=0.03)$, indicating the significantly different reductions

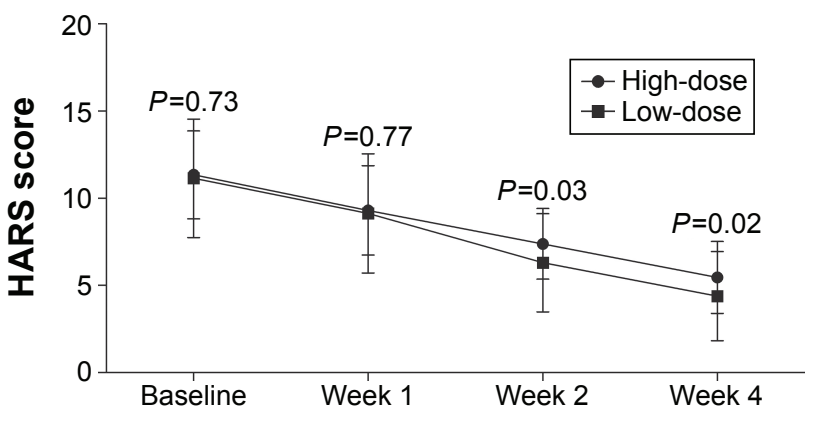

Figure 5 Average HARS scores at baseline and at weeks I, 2, and 4 in both groups.

Abbreviation: HARS, Hamilton Anxiety Rating Scale. between the two groups. The average HARS scores were similar at baseline $(P=0.73)$ and week $1(P=0.77)$, but were significantly different at week $2(P=0.03)$ and week 4 $(P=0.02)$ between the two groups.

\section{Discussion}

Previous study reported that low-dose betahistine alone was less effective than the high-dose betahistine in treating residual dizziness after successful CRP for BPPV.${ }^{18}$ Considering the good synergistic effect of CBT when used together with other treatment methods, we conducted this study to check whether the addition of CBT could make low-dose betahistine produce similar results to high-dose betahistine in treating residual dizziness. Our results showed that, after 4-week treatment, the duration of residual dizziness and average DHI score had no difference between the two groups. Meanwhile, compared to the high-dose betahistine, the lowdose betahistine plus CBT could yield significantly lower average HDRS score and HARS score. In addition, in the high-dose group, four patients withdrew due to the intolerable stomach upset, which might indicate that the low-dose betahistine (no patient experienced intolerable stomach upset) was more acceptable. Therefore, these results demonstrated that the addition of CBT could make low-dose betahistine produce similar results to high-dose betahistine in treating residual dizziness. Moreover, the low-dose betahistine plus CBT showed some advantages over high-dose betahistine in relieving depressive and anxiety symptoms and should be further explored.

Betahistine is an anti-vertigo medication, which is commonly prescribed for balance disorders or used to alleviate the vertigo symptoms of Ménière's disease. ${ }^{28}$ Previous studies found that it was also effective in the management of vertigo. ${ }^{29,30}$ As a weak agonist of the histamine $\mathrm{H} 1$ receptor and a strong antagonist of the histamine $\mathrm{H} 3$ receptor, ${ }^{31}$ betahistine has two main mechanisms of action. Primarily, as an agonist on the $\mathrm{H} 1$ receptors located on blood vessels in the inner ear, it could be helpful for local vasodilation and increase the permeability of capillaries. This could help to reverse or alleviate the underlying problem of endolymphatic hydrops. ${ }^{32}$ More importantly, the powerful antagonistic effects of betahistine at $\mathrm{H} 3$ receptors could increase the levels of serotonin, acetylcholine, neurotransmitter histamine, and norepinephrine released from the nerve endings. Then, the increased level of histamine releasing from the histaminergic nerve endings could stimulate the receptors, which well explains the potent vasodilatory effects of betahistine in the inner ear. ${ }^{33}$ 
CBT has been found to have the limited long-term effect on phobic postural vertigo ${ }^{20}$ and could yield significant improvements in dizziness-related symptoms. ${ }^{21}$ It is also often recommended to treat depression, which is a seriously debilitating mental disorder with unclear pathogenesis. ${ }^{34,35}$ Our previous study found that the addition of CBT could significantly improve the efficacy of sertraline in reducing the DHI score, HDRS score, and HARS score and reduce the dose of sertraline used. ${ }^{22}$ The previous study recommended the high-dose betahistine over low-dose betahistine in treating residual dizziness after successful CRP for BPPV. ${ }^{18}$ However, in this study, our results demonstrated that the low-dose betahistine plus CBT showed a nonsignificantly better efficacy than high-dose betahistine in treating residual dizziness. Moreover, no patient in the low-dose group experienced intolerable stomach upset. Previous study reported that the anxiety/depressive symptoms might also be the important causes of residual dizziness. ${ }^{15}$ Interestingly, our present results showed that the average HDRS and HARS scores were significantly lower in the low-dose group than in the high-dose group. Therefore, the CBT might enhance the efficacy of betahistine by improving the anxiety/depressive symptoms of patients.

Several limitations in our study should be mentioned. First, the sample size in each group was relatively small, limiting the available power and ability to find the more subtle changes; although we have found the significant improvements on the three measurement scales. Second, all patients with BPPV were from the same city, which possibly limits the general applicability of our conclusion. ${ }^{36,37}$ Third, the treatment was only continued for 4 weeks. The long-term effects of low-dose betahistine plus CBT were not assessed here. Fourth, no placebo control group was designed in this study, although previous study reported that the betahistine was significantly better than placebo in treating residual dizziness. ${ }^{18}$ Fifth, only the canalithiasis type of BPPV patients with posterior semicircular canal involvement were included. Whether or not our conclusion was appropriate for other types of BPPV patients needs further investigation. Sixth, we used symptoms to evaluate the efficacy of these two methods, but they had methodological limitations because they were subjective data. Future studies should use some objective indicators, such as slow phase velocity of positional nystagmus, to further evaluate the efficacy of these two methods on residual dizziness after successful CRP for BPPV. Finally, we only compared the efficacy of two different dosages of betahistine here; future studies should compare the relative effectiveness of other doses, which might help the clinicians to select the optimal dose for particular patients.

\section{Conclusion}

Our study demonstrated that the low-dose betahistine plus CBT should be recommended over high-dose betahistine in treating residual dizziness after successful CRP for BPPV. The addition of CBT could reduce the dose of betahistine used and then generate substantial cost savings to the individual and community. The present results could be helpful for the development of more effective therapy methods in treating residual dizziness over time. Limited by the relatively small sample size, our conclusion still needs future studies to validate and support.

\section{Disclosure}

The authors report no conflicts of interest in this work.

\section{References}

1. Furman JM, Cass SP. Benign paroxysmal positional vertigo. $N$ Engl J Med. 1999;341(21):1590-1596.

2. von Brevern M, Radtke A, Lezius F, et al. Epidemiology of benign paroxysmal positional vertigo: a population based study. J Neurol Neurosurg Psychiatry. 2007;78(7):710-715.

3. Bhattacharyya N, Baugh RF, Orvidas L, et al. Clinical practice guideline: benign paroxysmal positional vertigo. Otolaryngology-Head and Neck Surgery. 2008;139(5_suppl):47-81.

4. Baloh RW, Honrubia V, Jacobson K. Benign positional vertigo: clinical and oculographic features in 240 cases. Neurology. 1987;37(3): 371-378.

5. Lopez-Escamez JA, Gamiz MJ, Fernandez-Perez A, Gomez-Fiñana M. Long-term outcome and health-related quality of life in benign paroxysmal positional vertigo. Eur Arch Otorhinolaryngol. 2005;262(6): $507-511$.

6. Imai $\mathrm{T}$, Ito $\mathrm{M}$, Takeda $\mathrm{N}$, et al. Natural course of the remission of vertigo in patients with benign paroxysmal positional vertigo. Neurology. 2005;64(5):920-921.

7. Kerber KA, Burke JF, Skolarus LE, et al. Use of BPPV processes in emergency department dizziness presentations: a population-based study. Otolaryngol Head Neck Surg. 2013;148(3):425-430.

8. Helminski JO, Zee DS, Janssen I, Hain TC. Effectiveness of particle repositioning maneuvers in the treatment of benign paroxysmal positional vertigo: a systematic review. Phys Ther. 2010;90(5):663-678.

9. Tirelli G, Nicastro L, Gatto A, Tofanelli M. Repeated canalith repositioning procedure in BPPV: Effects on recurrence and dizziness prevention. Am J Otolaryngol. 2017;38(1):38-43.

10. Su P, Liu YC, Lin HC. Risk factors for the recurrence of postsemicircular canal benign paroxysmal positional vertigo after canalith repositioning. J Neurol. 2016;263(1):45-51.

11. Li JC, Jc L. Mastoid oscillation: a critical factor for success in canalith repositioning procedure. Otolaryngol Head Neck Surg. 1995;112(6): 670-675.

12. Kim HA, Lee H. Autonomic dysfunction as a possible cause of residual dizziness after successful treatment in benign paroxysmal positional vertigo. Clin Neurophysiol. 2014;125(3):608-614.

13. Mulavara AP, Cohen HS, Peters BT, Sangi-Haghpeykar H, Bloomberg JJ. New analyses of the sensory organization test compared to the clinical test of sensory integration and balance in patients with benign paroxysmal positional vertigo. Laryngoscope. 2013;123(9):2276-2280. 
14. Mandalà M, Santoro GP, Asprella Libonati G, et al. Double-blind randomized trial on short-term efficacy of the Semont maneuver for the treatment of posterior canal benign paroxysmal positional vertigo. J Neurol. 2012;259(5):882-885.

15. Jung HJ, Koo JW, Kim CS, Kim JS, Song JJ. Anxiolytics reduce residual dizziness after successful canalith repositioning maneuvers in benign paroxysmal positional vertigo. Acta Otolaryngol. 2012;132(3): 277-284.

16. Deng W, Yang C, Xiong M, Fu X, Lai H, Huang W. Danhong enhances recovery from residual dizziness after successful repositioning treatment in patients with benign paroxysmal positional vertigo. $\mathrm{Am} \mathrm{J}$ Otolaryngol. 2014;35(6):753-757.

17. Ge L, Han L, Huang X, Guan C. Betahistine was effective on residual dizziness after successful repositioning treatment in BPPV patients. Zhejiang Practical Medicine. 2015;20(5):329-331.

18. Ma DJ, Hu J, Lu X, Xu JQ. Efficacy observation of different doses of betahistine in the treatment of benign paroxysmal positional vertigo with residual dizziness. Chin J of Clinical Rational Drug Use. 2018;11(5A):23-24.

19. Benjamin CL, Puleo CM, Settipani CA, et al. History of cognitivebehavioral therapy in youth. Child Adolesc Psychiatr Clin N Am. 2011; 20(2):179-189.

20. Holmberg J, Karlberg M, Harlacher U, Magnusson M. One-year follow-up of cognitive behavioral therapy for phobic postural vertigo. J Neurol. 2007;254(9):1189-1192.

21. Edelman S, Mahoney AE, Cremer PD. Cognitive behavior therapy for chronic subjective dizziness: a randomized, controlled trial. Am J Otolaryngol. 2012;33(4):395-401.

22. Yu YC, Xue H, Zhang YX, Zhou J. Cognitive behavior therapy as augmentation for sertraline in treating patients with persistent posturalperceptual dizziness. Biomed Res Int. 2018;2018:8518631.

23. Parnes LS, Agrawal SK, Atlas J. Diagnosis and management of benign paroxysmal positional vertigo (BPPV). CMAJ. 2003;169(7):681-693.

24. Otorhinolaryngology Branch of Chinese Medical Association. Diagnosis and efficacy evaluation of benign paroxysmal positional vertigo. Chin J Otorhinolaryngol Head Neck Surg. 2007;42(3):163-164.

25. Epley JM. The canalith repositioning procedure: for treatment of benign paroxysmal positional vertigo. Otolaryngol Head Neck Surg. 1992;107(3):399-404.
26. Jacobson GP, Newman CW. The development of the dizziness handicap inventory. Arch Otolaryngol Head Neck Surg. 1990;116(4):424-427.

27. Che T, Teng X, Huang Q, et al. Agomelatine versus fluoxetine in glycemic control and treating depressive and anxiety symptoms in type 2 diabetes mellitus subjects: a single-blind randomized controlled trial. Neuropsychiatr Dis Treat. 2018;14:1527-1533.

28. Lacour M, van de Heyning PH, Novotny M, Tighilet B. Betahistine in the treatment of Ménière's disease. Neuropsychiatr Dis Treat. 2007; 3(4):429-440.

29. Ding X, Gu H, Luo J, Li J. Clinical observation of betahistine in the treatment of 80 cases of vertigo. Mod Diagn Treat. 2013;24(15): 3433-3434.

30. Guo K. Therapeutic effect of betahistine on clinical symptoms and adverse events in patients with vertigo. Medical Theory and Practice. 2018;31(4):521-522.

31. Jeck-Thole S, Wagner W. Betahistine. Drug Safety. 2006;29(11): 1049-1059.

32. Bertlich M, Ihler F, Weiss BG, et al. Role of capillary pericytes and precapillary arterioles in the vascular mechanism of betahistine in a guinea pig inner ear model. Life Sci. 2017;187:17-21.

33. Møller MN, Kirkeby S, Vikeså J, Nielsen FC, Caye-Thomasen P. Expression of histamine receptors in the human endolymphatic sac: the molecular rationale for betahistine use in Menieres disease. Eur Arch Otorhinolaryngol. 2016;273(7):1705-1710.

34. Mata DA, Ramos MA, Bansal N, et al. Prevalence of depression and depressive symptoms among resident physicians: a systematic review and meta-analysis. JAMA. 2015;314(22):2373-2383.

35. Chen JJ, Zheng P, Liu YY, et al. Sex differences in gut microbiota in patients with major depressive disorder. Neuropsychiatr Dis Treat. 2018;14:647-655

36. Lin L, Chen XM, Liu RH. Novel urinary metabolite signature for diagnosing postpartum depression. Neuropsychiatr Dis Treat. 2017; 13:1263-1270.

37. Chen JJ, Bai SJ, Li WW, et al. Urinary biomarker panel for diagnosing patients with depression and anxiety disorders. Transl Psychiatry. 2018;8(1):192
Neuropsychiatric Disease and Treatment

\section{Publish your work in this journal}

Neuropsychiatric Disease and Treatment is an international, peerreviewed journal of clinical therapeutics and pharmacology focusing on concise rapid reporting of clinical or pre-clinical studies on a range of neuropsychiatric and neurological disorders. This journal is indexed on PubMed Central, the 'PsycINFO' database and CAS,

\section{Dovepress}

and is the official journal of The International Neuropsychiatric Association (INA). The manuscript management system is completely online and includes a very quick and fair peer-review system, which is all easy to use. Visit http://www.dovepress.com/testimonials.php to read real quotes from published authors. 